\title{
Outpatient combined intracavitary and interstitial cervical brachytherapy: barriers and solutions to implementation of a successful programme - a single institutional experience
}

\author{
Poh Wee Tan, MD, Vicky Y. Koh, MD, FRANZCR, Johann I. Tang, MD, FRANZCR \\ Department of Radiation Oncology, National University Cancer Institute Singapore, Singapore
}

\begin{abstract}
Involvement of parametrial disease in locally advanced cervical patients poses a challenge for women undergoing brachytherapy. Current use of the Fletcher suit applicator may not adequately cover the high risk clinical target volume (HR CTV), especially in the parametrial region due to the physical qualities of brachytherapy from the inverse square law and the need to respect organs at risk (OAR) constraints, and leads to lower local control rates. Combined intracavitary and interstitial brachytherapy with the use of 1 or 2 interstitial needles allows adequate coverage of the HR CTV and the clinical evidence have demonstrated a correlation with better clinical results. This procedure is often resource intensive, requiring inpatient stay and magnetic resonance imaging (MRI) planning. In departments where such resources are limited, there is a poor uptake of interstitial brachytherapy. This article discusses the technique of combined intracavitary and interstitial brachytherapy in an outpatient setting, and explores the issues and barriers for implementation and suggestions to overcome such barriers.
\end{abstract}

Key words: brachytherapy, cervical cancer, interstitial, outpatient, paracervical block.

\section{Purpose}

Concurrent chemoradiotherapy is the standard treatment for locally advanced cervical cancer [1-4]. Standard treatment for radiotherapy usually involves the use of external beam radiotherapy followed by 3 channel intracavitary brachytherapy, using the 3 channel Fletcher Suite applicator (Elekta AB, Stockholm, Sweden) given the higher survival rates $[5,6]$. For tumors involving the parametrium or pelvic side wall, 3 channel Fletcher suite applicator may not provide adequate dosimetric coverage of the tumor, and hence lead to higher recurrence rate [7]. Combined intracavitary and interstitial cervical brachytherapy, using either the Vienna Ring applicator (Elekta AB, Stockholm, Sweden) or Utrecht applicator (Elekta AB, Stockholm, Sweden) plus interstitial needles placed in the parametrium area has shown to improved tumor dosimetric coverage, leading to higher local control as compared to non interstitial treatment $[8,9]$. As this procedure is done in an operating theatre (OT) setting requiring the use of general or epidural anaesthesia, access to such resources is limited. To overcome the OT access issue, outpatient combined intracavitary and interstitial cervical brachytherapy is an attractive option. The aim of this article is to share and discuss the use of combined intracavitary and interstitial cervical brachytherapy in an outpatient setting at the author's institution, and explores the issues and barriers for implementation and suggestions to overcome such barriers.

\section{Rationale for combined intracavitary and interstitial treatment}

Results from the Vienna group has demonstrated that to have effective local control, the minimal dose to the high risk clinical target volume (HR CTV) should be greater or equal than 87 Gy [10]. Without the use of interstitial needles, such dose to the HR CTV is difficult to achieve due to the adjacent bowel and rectal anatomy, limiting the HR CTV to below $87 \mathrm{~Gy}$. However, with interstitial needles in place, one is able to achieve the HR CTV should be greater
Address for correspondence: Johann I. Tang, MD, FRANZCR, Department of Radiation Oncology, National University Cancer Institute Singapore, 1E Kent Ridge Road, 119228 Singapore, Singapore, phone: +65 67724869, ® e-mail: johann_tang@nuhs.edu.sg
Received: 05.04 .2015

Accepted: 14.06 .2015

Published: 25.06.2015 

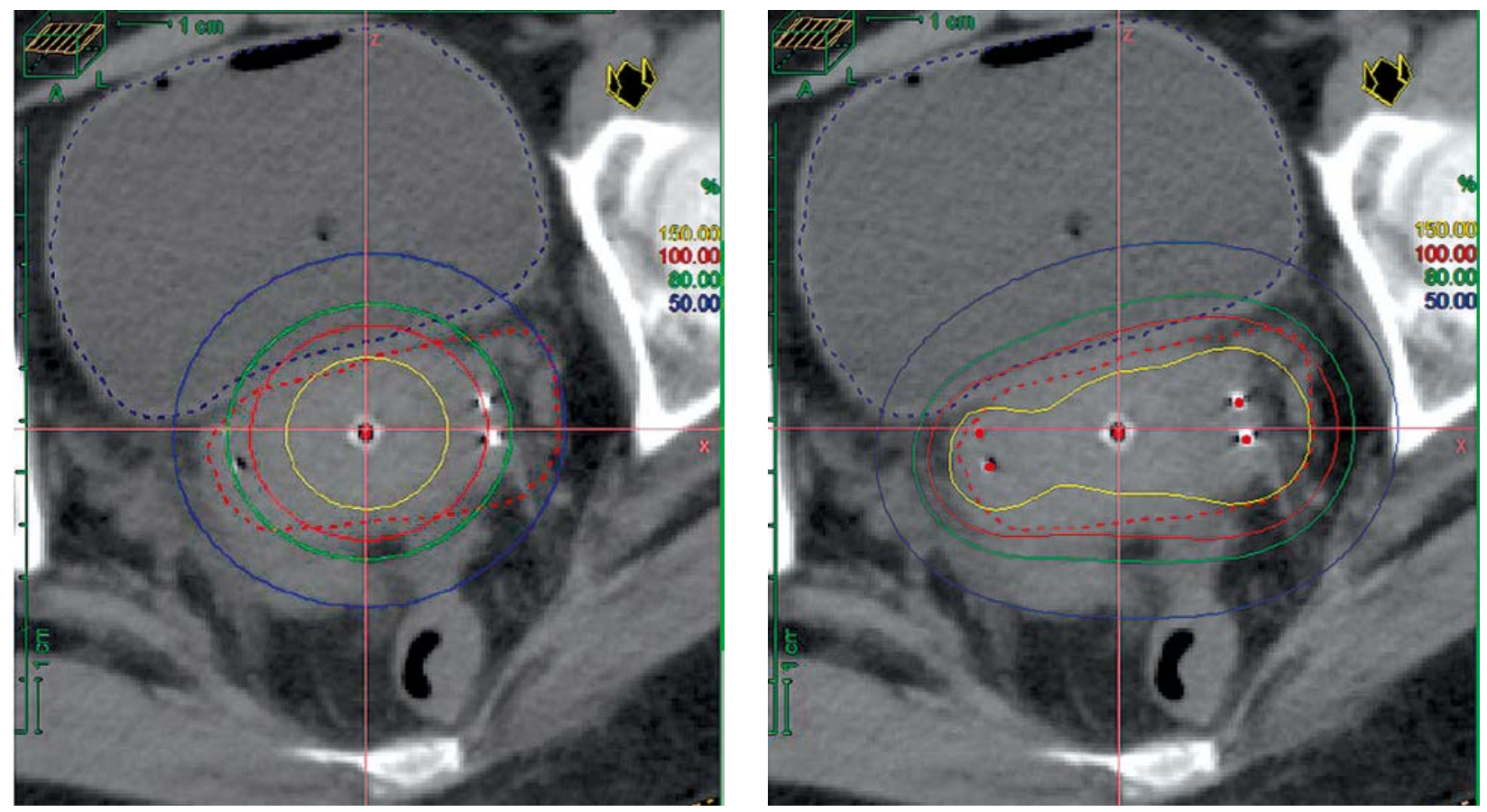

Fig. 1. Patients with bilateral parametrium disease benefit the greatest from interstitial brachytherapy

or equal than $87 \mathrm{~Gy}$, yet respecting the organ at risk dose of the adjacent normal tissues [8-10].

\section{Patient selection}

Patients with cervical stage $2 \mathrm{~B}$ and stage $3 \mathrm{~B}$ with parametrial residual disease post chemotherapy and external beam radiotherapy would benefit the most from interstitial cervical brachytherapy, especially for patients with residual bilateral parametrial disease [10] (Figure 1). Compared to 3D Fletcher suit applicator, the greatest advantage of combined intracavitary and interstitial brachytherapy is the superior dosimetric coverage of the HR CTV, allowing for better therapeutic ratio [8-10]. This permits the HR CTV to be above $87 \mathrm{~Gy}$, thus improving local control rates. This form of adaptive brachytherapy is personalized to each individual patient's anatomy in order to lead to the best results. Other patient selection factors include fitness to undergo anaesthesia, suitable pelvic anatomy, and tumor topography for appropriate applicator placement, as well as availability to preplanning clinical examination and imaging findings.

\section{Method of insertion}

The entire radiotherapy treatment should be completed within 7 weeks, hence the brachytherapy should ideally commence at the end of external beam radiotherapy and should be avoided with concurrent chemotherapy [11-13]. With regards to the brachytherapy dose, there is a range of doses used as recommended by the American Brachytherapy Society guidelines but at our institution, our brachytherapy treatment schema is 7 Gy $\times 4$ fractions, treating a maximum of 2 fractions a week with at least 8 hours in between fractions [12,13]. Two to 3 days prior to the procedure, a pre-brachytherapy planning magnetic resonance imaging (MRI) scan is performed to evaluate the extend of the parametrium, identification of important normal tissue structures such as the uterine arteries, as well as to determine where to place the interstitial needles to achieve optimal coverage [14,15]. Consultation with the radiologist may be useful in aiding the pre-brachytherapy planning.

Twenty-four hours prior to the procedure, oral fleet is given to the patient to ensure adequate bowel preparation. By reducing the chance of fecal material or flatus in the bowel, this reduces the likelihood of the bowel being adjacent to the HR CTV region [12]. On the day of the procedure, the patient is fasted 6 hours before to allow for moderate sedation using a combination of oxycodone $5 \mathrm{mg}$ capsules and midazolam given by the anesthetist. After positioning the patient in a lithotomy position, a formal examination of the cervix and the parametrium and a per rectum is done digitally to assess the extent of the tumor. With the speculum in place, a paracervical block is then performed, injecting $2 \%$ lignocaine into the $2,4,8,10$ o'clock positions in the cervical parametrium area (Figure 2). This is to anaesthetize the adjacent area to facilitate the insertion of the interstitial needles in the parametrium [16].

Once the Utrecht applicator is in situ, the planning of where to insert and the length of the needle insertion is determined from the pre-brachytherapy MRI, avoiding important anatomical structures such as the uterine artery. Once determined, usually 1-2 needles are inserted into the parametrium. The vaginal is then packed with gauze and the applicator secured, and the patient is then transferred to the computed tomography (CT) scanner. 
Prior to the CT scan, the bladder is filled with $200 \mathrm{mls}$ of normal saline to facilitate the movement of the small bowel away from the HR CTV region [17]; $5 \mathrm{~mm}$ CT scan of the abdo-pelvis area is then acquired. At this juncture, the radiation oncologist should review the location and depth of needle insertion, as well as any adjustment to the length of the needles is then done and the CT scan reacquired once more till the depth of needles adequately covers the HR CTV region.

The HR CTV and the various organs at risk (OAR) are then contoured as per the Groupe Européen de $\mathrm{Cu}$ rietherapie (GEC) and the European Society for Radiotherapy \& Oncology (GEC-ESTRO) protocol $[18,19]$. The prescription will be $7 \mathrm{~Gy} \times 4$ fractions. The various OAR recommendations are listed in Table $1[16,20]$.

\section{Discussion}

Combined intracavitary and interstitial brachytherapy has been clinically proven to show better dosimetric coverage, leading to higher local control rates. However, the uptake of this procedure remains low despite these excellent results. Barriers to combined intracavitary and interstitial brachytherapy implementation can be summed up into doctor related, patient related, and procedure related [21].

Doctor related barriers are mainly due to inadequate training in the use of combined intracavitary and interstitial cervical brachytherapy devices, and being unfamiliar with contouring using the GEC-ESTRO protocol. To encourage upgrading of clinical skills and continued medical education, European society of therapeutic radiation oncology (ESTRO) has setup a teaching school with annual courses on combined intracavitary and interstitial gynaeoncology brachytherapy including mix of didactic lectures, contouring sessions, and discussion on clinically challenging cases, which the author found beneficial, useful, and highly recommended to readers interested in setting up a interstitial gynaeoncology program [22]. In addition, International Atomic Energy and Agency (IAEA) and BrachyAcademy (Elekta) also conduct similar educational courses, focusing more on implementation issues and clinical hands on sessions [23,24].

Patient related barriers are few and are concentrating on poor communication or a lack of knowledge of why interstitial brachytherapy is indicated or needle phobia. With clear and concise communication during the informed consent process, as well as involving the patient by explaining the insertion process using the pre-planning MRI scan, the author's experience suggest that most patients are generally less fearful and more inclined to undergo this procedure. One suggestion by the author is to explain the procedure using a power point presentation, detailing the insertion process, evidence of why it is better than Fletcher suite applicator, and the clinical results of the interstitial experience with associated potential side effects of this procedure helps patient to understand the procedure better and are hence less apprehensive or less needle phobic.

Procedure related barriers include the lack of access to MRI scanners on the implant day or the limited access to

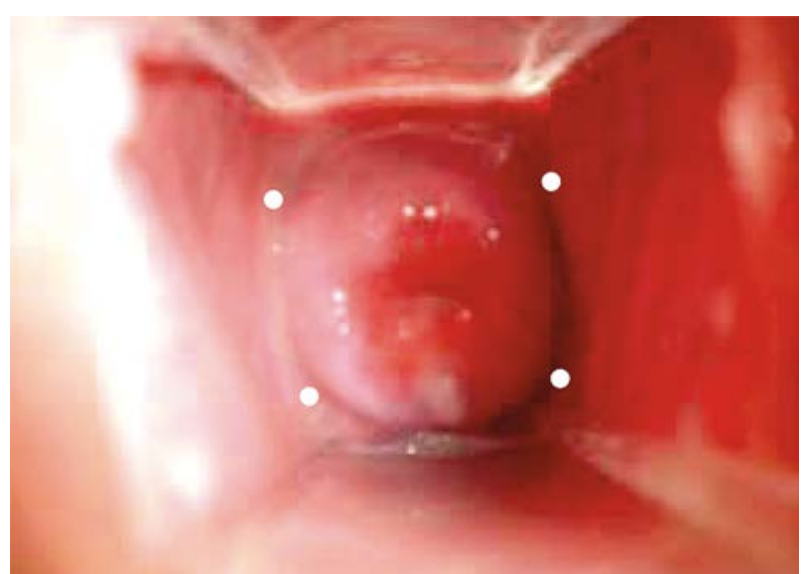

Fig. 2. Paracervical block. Paracervical anaesthetic block is placed at the 2, 4, 8, 10 o' clock position in the cervical parametrium area as denoted by the white dots

Table 1. Prescription dose and organs at risk values used at our centre

\begin{tabular}{l} 
Prescription dose: \\
\hline EBRT: 50.4 Gy in 28 fractions \\
PDR: 7 Gy $\times 4$ fractions \\
Point A dose to be reported \\
\hline RR CTV: $D_{90} \geq 87$ Gy \\
\hline Rectal $D_{2 c c} \leq 75$ Gy \\
\hline Bladder $D_{2 c c}: \leq 100$ Gy
\end{tabular}

OT resources and issue of adequate regional anaesthesia if done as outpatient.

\section{MRI versus CT scan for planning}

Currently, the gold standard for combined intracavitary and interstitial is MRI planning $[25,26]$. However, for most radiotherapy centres in the worlds, access to MRI scanners is an issue due to resource limitation [27]. However, most radiotherapy centres have a CT scanner in house, which is used for everyday radiotherapy simulation planning process. To better correlate MRI planning using CT scans, a suggestion is to perform a pre brachytherapy insertion MRI 2-3 days before the actual insertion day. This pre insertion MRI is crucial as it allows the radiation oncologist to determine the extent of the parametrial disease, placement of interstitial needles, as well as location of important anatomical structure such as the uterine arteries to avoid [28].

Alternatively, MRI could be obtained as a first fraction with subsequent fractions using CT scans [13]. Conclusions from the Vienna group using this method showed that this combination method is feasible and best used for small tumours with minimal parametrial involvement only. For tumors with higher volume of residual disease in the parametrium, the authors recommend avoiding such a technique. 
In addition, there are published guidelines on using CT scans for contouring regions of interest [29-32]. Studies evaluating CT scan contouring have suggested that with good preplanning using information from clinical examination of the cervix and the use of the pre insertion MRI scan, there is good correlation between MRI and CT scan for contouring [33-35]. This would facilitate more centres adopting combined intracavitary and interstitial brachytherapy using CT scans for contouring.

\section{Outpatient versus inpatient}

A major resource limitation at the author's centre is the access to OT facilities. At the author's centre, insertion of the Fletcher suite applicator is done in the radiotherapy department under moderate sedation on an outpatient basis. Given that the Utrecht applicator insertion is similar to the 3 channel applicator insertion except for the interstitial component, this too may be done as an outpatient procedure. To minimize pain and discomfort, moderate sedation together with a paracervical block is used to anaesthesize the patient to provide better pain relief. At our centre, we have successfully treated patients in an outpatient centre using combination of moderate anaesthesia with paracervical block, and have found the procedure well tolerated with minimal post procedure complications, which is comparable to other radiotherapy centres $[33,34]$. Hence, in the author's own experience, outpatient combined intracavitary and interstitial cervical brachytherapy may be a safe and feasible option when done together with pre insertion MRI brachytherapy planning and should be advocated.

Additional advantages in an outpatient setting include no need to compete for inpatient beds with other medical disciplines, lower risk of hospital acquired infection, lower financial cost to patient, and better workflow protocol within the department.

\section{Anaesthesia: locoregional blocks with moderate sedation versus general anaesthesia}

Regional anaesthesia, using a paracervical block is a simple and effective method to provide adequate anaesthesia around the uterus, cervix, and parametrial region. Under visual inspection, $2 \%$ of lignocaine solution is injected into the paracervical region creating the anaesthesia block. Advantage of lignocaine is that it is a safe and fast acting with a short duration of anaesthesia (about 1 hour), compared to Bupivacaine, which is known to cause life threatening cardiotoxicity. If longer anaesthesia is required, safer alternatives to Bupivacaine include Ropivacaine and Chirocaine; both having safer cardiotoxicity profiles compared to Bupivacaine. Complications of administering such a block include risk of bleeding, iatrogenic infection, and intravascular injection. In the author's centre, the learning curve for administration of such a block is about 5 patients done under supervision before one becomes competent. Advantages of the paracervical block include avoiding an epidural or general anaesthesia, which generally carries a higher complication risk [35].

\section{Conclusions}

Combined intracavitary and interstitial cervical brachytherapy should be the standard of care for patients with residual parametrial disease post chemotherapy and external beam radiotherapy, given the superior dosimetric coverage translating into higher local control rates. Ideally, outpatient insertion should be the preferred method and suggestions to overcome such barriers in an outpatient setting have been discussed. Sharing of clinical experience from other centres adopting outpatient combined intracavitary and interstitial cervical treatment is needed to validate this procedure as an outpatient procedure.

\section{Disclosure}

Authors report no conflict of interest.

\section{References}

1. Rose PG, Ali S, Watkins E et al. Long-term follow-up of a randomized trial comparing concurrent single agent cisplatin, cisplatin- based combination chemotherapy, or hydroxyurea during pelvic irradiation for locally advanced cervical cancer: a Gynecologic Oncology Group Study. J Clin Oncol 2007; 25: 2804-2810.

2. Whitney CW, Sause W, Bundy BN et al. Randomized comparison of fluorouracil plus cisplatin versus hydroxyurea as an adjunct to radiation therapy in stage IIB-IVA carcinoma of the cervix with negative para-aortic lymph nodes: a Gynecologic Oncology Group and Southwest Oncology Group study. J Clin Oncol 1999; 17: 1339-1348.

3. Thomas GM. Improved treatment for cervical cancer - concurrent chemotherapy and radiotherapy. N Engl J Med 1999; 340: 1198-1200.

4. Peters WA, Liu PY, Barrett RJ et al. Concurrent chemotherapy and pelvic radiation therapy compared with pelvic radiation therapy alone as adjuvant therapy after radical surgery in high-risk early-stage cancer of the cervix. J Clin Oncol 2000; 18: 1606-1613.

5. Tanderup K, Eifel PJ, Yashar CM et al. Curative therapy for locally advanced cervical cancer: Brachytherapy is NOT optional. Int J Radiat Oncol Biol Phys 2014; 88: 537-539.

6. Han K, Milosevic M, Fyles A et al. Trends in the utilization of brachytherapy in cervical cancer in the United States. Int J Radiat Oncol Biol Phys 2013; 87: 111-119.

7. Mohamed S, Kallehauge J, Fokdal L et al. Parametrial boosting in locally advanced cervical cancer: combined intracavitary/ interstitial brachytherapy vs. intracavitary brachytherapy plus external beam radiotherapy. Brachytherapy 2015; 14: 23-28.

8. Pötter R, Georg P, Dimopoulos JC et al. Clinical outcome of protocol based image (MRI) guided adaptive brachytherapy combined with 3D conformal radiotherapy with or without chemotherapy in patients with locally advanced cervical cancer. Radiother Oncol 2011; 100: 116-123.

9. Pötter R, Dimopoulos J, Georg P et al. Clinical impact of MRI assisted dose volume adaptation and dose escalation in brachytherapy of locally advanced cervix cancer. Radiother Oncol 2007; 83: 148-155.

10. Dimopoulos JC, Pötter R, Lang S et al. Dose-effect relationship for local control of cervical cancer by magnetic resonance image-guided brachytherapy. Radiother Oncol 2009; 93: 311-315.

11. Perez CA, Grigsby PW, Castro-Vita et al. Carcinoma of the uterine cervix. Impact of prolongation of overall treatment 
time and timing of brachytherapy on outcome of radiation therapy. Int J Radiat Oncol Biol Phys 1995; 32: 1275-1288.

12. http://www.americanbrachytherapy.org/guidelines/cervical_cancer_task group.pdf. Accessed on $30^{\text {th }}$ March 2015.

13. https://www.embracestudy.dk/AboutProtocolDownload. aspx. Accessed on 30 ${ }^{\text {th }}$ March 2015.

14. Nesvacil N, Pötter R, Sturdza A et al. Adaptive image guided brachytherapy for cervical cancer: a combined MRI-/CTplanning technique with MRI only at first fraction. Radiother Oncol 2013; 107: 75-81.

15. http://www.nccn.org/professionals/physician_gls/pdf/ cervical.pdf. Accessed on $30^{\text {th }}$ March 2015.

16. Petric $P$, Hudej R, Hanuna $O$ et al. MRI-assisted cervix cancer brachytherapy pre-planning, based on application in paracervical anaesthesia: final report. Radiol Oncol 2014; 48: 293-300.

17. Georg P, Lang S, Dimopoulos JC et al. Dose-volume histogram parameters and late side effects in magnetic resonance image-guided adaptive cervical cancer brachytherapy. Int J Radiat Oncol Biol Phys 2011; 79: 356-362.

18. Haie-Meder C, Pötter R, Van Limbergen E et al. Recommendations from Gynaecological (GYN) GEC-ESTRO Working Group (I): concepts and terms in 3D image based 3D treatment planning in cervix cancer brachytherapy with emphasis on MRI assessment of GTV and CTV. Radiother Oncol 2005; 74: 235-245.

19. Pötter R, Haie-Meder C, Van Limbergen E et al. Recommendations from gynaecological (GYN) GEC ESTRO working group (II): concepts and terms in 3D image-based treatment planning in cervix cancer brachytherapy-3D dose volume parameters and aspects of 3D image-based anatomy, radiation physics, radiobiology. Radiother Oncol 2006; 78: 67-77.

20. Georg P, Pötter R, Georg D et al. Dose effect relationship for late side effects of the rectum and urinary bladder in magnetic resonance image-guided adaptive cervix cancer brachytherapy. Int J Radiat Oncol Biol Phys 2012; 82: 653-657.

21. Petric P, Viswanathan AN. Gynecologic Radiation Therapy. Springer Verlag, Berlin 2011.

22. http://www.estro.org/school. Accessed on $30^{\text {th }}$ March 2015.

23. http://www.iaea.org/technicalcooperation/documents/ Region-Announce-Docs/Asia-and-the-Pacific/2014-Aug/ RCA-TC.pdf. Access on 30 $0^{\text {th }}$ March 2015.

24. http://www.brachyacademy.com. Access on $30^{\text {th }}$ March 2015.

25. Dimopoulos JC, Petrow P, Tanderup K et al. Recommendations from Gynaecological (GYN) GEC-ESTRO Working Group (IV): Basic principles and parameters for MR imaging within the frame of image based adaptive cervix cancer brachytherapy. Radiother Oncol 2012; 103: 113-122.

26. Petric $P$, Mohammed-Al-Hammadi N. MRI findings at image guided adaptive cervix cancer brachytherapy: radiation oncologist's perspective. J Contemp Brachytherapy 2014; 6: 215-222.

27. Tanderup K, Viswanathan AN, Kirisits C et al. Magnetic resonance image guided brachytherapy. Semin Radiat Oncol 2014; 24: 181-191.

28. Dimopoulos JC, Schard G, Berger D et al. Systematic evaluation of MRI findings in different stages of treatment of cervical cancer: potential of MRI on delineation of target, pathoanatomic structures, and organs at risk. Int J Radiat Oncol Biol Phys 2006; 64: 1380-1388.

29. Viswanathan AN, Erickson B, Gaffney DK et al. Comparison and consensus guidelines for delineation of clinical target volume for CT- and MR-based brachytherapy in locally advanced cervical cancer. Int J Radiat Oncol Biol Phys 2014; 90: 320-328.

30. Lee LJ1, Damato AL, Viswanathan AN. Clinical outcomes of high-dose-rate interstitial gynecologic brachytherapy using real-time CT guidance. Brachytherapy 2013; 12: 303-310.
31. Kannan N1, Beriwal S, Kim H et al. High-dose-rate interstitial computed tomography-based brachytherapy for the treatment of cervical cancer: early results. Brachytherapy 2012; 11: 408-412.

32. Saitoh J, Ohno T, Sakurai H et al. High-dose-rate interstitial brachytherapy with computed tomography-based treatment planning for patients with locally advanced uterine cervical carcinoma. J Radiat Res 2011; 52: 490-495.

33. Dyk PT, Richardson S, Badiyan SN et al. Outpatient-based high-dose-rate interstitial brachytherapy for gynecologic malignancies. Brachytherapy 2015; 14: 231-237.

34. Bernstein M, Yaparpalvi R, Kuo H et al. CT-Guidance Allows Interstitial Implantation in an Outpatient Setting for Cervical Cancer Patients. Pract Radiat Oncol 2013; 3 (2 Suppl 1): S1.

35. Lim KH, Lu JJ, Wynne CJ et al. A study of complications arising from different methods of anesthesia used in high-doserate brachytherapy for cervical cancer. Am J Clin Oncol 2004; 27: 449-451. 